\title{
Theoretical and Practical Aspects of Integrating the Computer and Music Technologies into The Instrumental Performance Training of The Pedagogical University Students
}

\author{
Valery O. Malaschenko ${ }^{1 *}$, Marina A. Antonova ${ }^{1}$, Galina L. Knyazeva ${ }^{1}$, Igor A. Belokon ${ }^{1}$, \\ Boris A. Pechersky ${ }^{1}$
}

${ }^{1}$ Moscow City University, Department of Musical Art, Moscow, Russia

\begin{abstract}
The work reveals the general social and technological prerequisites for integrating computer technologies into Russian education at various levels and profiles of educational institutions. The reasons for the basic contradictions in traditional and innovative pedagogical principles in professional training of performing musicians have been substantiated. Productive ways to solve the problem have been described based on the leading developments of education researchers in the computer and music technologies as well as in electronic music. The essence of the computer and music technologies concept as a specialized field of music pedagogy in Russia has been revealed. Features of hardware and software have been defined based on the digital interface of musical instruments and the prospects for their use in pedagogical activities. The methods, forms, and conditions for practical implementation of experimental training of pianist students from the Institute of Culture and Arts of the Moscow City University have been presented. The generalized results and conclusions of the experimental training in instrument performance of the pedagogical university students using the software and hardware components of the computer and music technologies have been analyzed and discussed.
\end{abstract}

\section{Introduction}

The integration of a wide range of digital technologies into educational processes of various levels and specialties is an active and quickly evolving trend of holistic modernization of the education system. This state is a natural world phenomenon that actualizes the demands of modern civilized society as a whole [1].

In Russia's educational space, the system integration of the latest software and hardware technologies is rapidly growing. It is important to emphasize vocational education in Russian colleges and universities, where there is a need for additional reorientation of experts taking into account the prospects of practical interaction with the advanced technologies $[1,2,3]$. A similar situation is natural in all professional areas, and even in those with no direct need to interact with such technologies. An example is the field of classical art.

* Corresponding author: MalashenkoVO@mgpu.ru 
The fields of music and visual art illustrate the natural creative identity and fragility in relation to the possibilities of modern multimedia technologies which massively dominate in the virtual digital space [4]. However, the positive aspects of introducing new technologies into the field of art cannot be denied. New and fresh trends are emerging in the virtualization of the audio and visual art [5]. Nonstandard synthetic and syncretic creative interaction between humans and artificial intelligence appears. As a result, modelling of the balanced mechanisms of technology integration into the art, including at the modern education level, is reasonable $[1,6]$.

\section{Theoretical and methodological aspects}

Classical music pedagogy of Russia has a centuries-old experience in the training of performers and teachers. Its key principles have proven to be highly effective. In educating professionals of various specialties, teachers rely on centuries-old traditions of classical musical performance, the preservation of which is an important task of art and education. However, in educating professional musicians focused on contemporary music and mass culture, conservative pedagogical principles will require a system addition $[7,8]$. The additions should be formed based on a balanced synthesis of a wide range of innovations in recent decades.

First of all, it concerns the extent to which modern specialized technologies massively integrate into the training of performing musicians. These include electronic musical instruments, specialized software, and sound equipment. These technologies are homogeneous and are based on the musical instrument digital interface - MIDI [7].

MIDI technologies are a set of hardware, technical, and software solutions running on a unified digital communication protocol created exclusively for the professional music and performance activities [7,9]. MIDI-coding combined with a sequencer is an alternative digital music writing that helps to expand the creative tools of musicians of various specialties. Consequently, the learning of MIDI technologies is inseparable when teaching modern music performance.

In the Russian music education, the basis for the formation of these areas of scientific and educational activity was laid at the end of the 20th century [10,11]. Since the beginning of the 21 st century, such areas of music pedagogy as computer and music technology and electronic music instruments have been successfully established in the Russian educational paradigm. The leading Russian education researchers in this field are I. M. Krasilnikov, I. B. Gorbunova, A. Cameris, etc. In the course of their activities, basic principles and theoretical and methodical materials were developed $[11,12]$.

Currently, the study of modern computer programs and the teaching of electronic musical instruments are successfully practiced in art and pedagogical education, at the music schools, pedagogical colleges, and in the universities $[10,11,12]$.

A different situation is taking place in creative colleges and universities. The teaching staff of creative universities is ambivalent about the integration of these areas into the academic and performance training of students. Many educators see the risks of suppression of the performance culture on classical acoustic musical instruments in such trends. In-depth study of the features of these technologies is carried out only in the framework of the training of sound engineers and composers.

The latest pedagogical developments in modeling an optimal trajectory of modern instrument performance development in the context of advances in the software and hardware MIDI technologies are a promising task. The Russian pedagogical community and pedagogical universities in general play a leading role in solving such problems.

Currently, active educational and methodical developments on the integration of the computer and music technologies and electronic music tools into educational training are in 
progress in several Russian pedagogical universities. The practical implementation of the bachelor's and master's degree programs at the Herzen University has begun based on the academic "computer and music technology" laboratory (St. Petersburg) [10, 12]. Similar educational areas have begun to be implemented in the Institute of Culture and Arts of the Moscow City University, the RAE Institute of Arts Education, and other pedagogical universities in Russia [12, 13, 14, 15].

\section{Experimental Learning Implementation}

This work describes the main results of the pedagogical activities on the integration of the computer and music technologies and electronic musical instruments into the training of the music and education students of the Department of Musical Arts of the Institute of Culture and Arts of the Moscow City University, which have been implementing from 2015 till present $[15,16,17]$.

The experimental learning process was based on exploring the possibilities of the modern software and hardware components based on the musical instruments digital interface.

Initially, the students were learning modern profile software. The interface and capabilities of computer programs of digital audio workstations, virtual instruments, and effects were studied.

In the learning of digital audio workstation programs, musical arrangements were gradually created. Music text served as a base. During the transfer of music information to the program, the students adapted to the virtual sequencer format. The activities were monitored in programs by ear in real time. The piece of music transferred into the program was arranged, by separating the texture range and duplication of voices. Virtual instruments such as synthesizers and samplers were used. Individual tracks of virtual instruments were sound-processed using virtual effects, and the work was carried out on the overall sound design of the arrangement. Further, the arrangement was converted into sound formats. This form of creative activity contributed to the natural learning of digital audio workstations and virtual instruments in practice.

In the future, creative work was carried out at a more complex level. For students the task was to recreate the arrangement by ear as close as possible to the original one. Only sound recordings of different timbre repertories and styles were used as the source material. The music text of the pieces was not used. In the implementation, the same software tools as in the work with music text were used. The high complexity of the task allowed to distribute its implementation into several classes.

Practical activities of the students to learn the computer and music technologies were carried out in a computer class. The students used digital audio workstations (Cubase, FL Studio, Reaper) and virtual instruments (NIFM 8, NI Kontakt, and Nexus). Each student's workplace was equipped with a MIDI keyboard and closed back headphones.

Later, the hybrid application of various keyboards in conjunction with computer programs was tested. The key tasks were to identify optimized combinations of software and hardware that contributed to the productive implementation of the students' music performance.

During the experiments, the pianist students performed piano and organ pieces on various electronic keyboards, such as synthesizers, digital pianos, and digital organs. Several keyboards were combined and the organ design was partially simulated. Electronic keyboards used both the timbres close to the acoustic instruments and those with bright specific electronic sound.

Both classical works of Baroque composers, Viennese classics and impressionists, as well as jazz pieces and contemporary music of the 20th century were used as a repertoire for experimental musical performance. The pieces selection criteria were modal and tonal 
repertories, metrorhythmic structure, degree of diversity and complexity of texture, and the ability to adapt to electronic and simulation timbres.

Recordings of concert performances and electro-acoustic pieces by K. Schulze, JeanMichel Jarre, E. Artemyev, C. Korea, J. Rudess and others were used as additional samples for the analysis of electronic musical performance. The analysis of the creative and performance activities of these electronic music artists revealed special authentic types of timbres of classical analog synthesizers, variations of their combinations, and sound processing techniques.

Computer programs and virtual musical instruments (VST) were used as a supplement to the hardware instruments in the performance. The performance on them was possible by connecting electronic keyboards to a computer used as a sound module. Additional sound equipment like audio board, compressor, and active acoustic systems were also used.

These types of practical activities with the pianist students were carried out in the individual instrumental cycle disciplines. The forms of performing activities included solo and ensemble performance on musical instruments. The students' performance activities took place in the chamber concert hall.

\section{Results}

The generalized results of the students' practice in the learning of the MIDI software and hardware components revealed the high efficiency of parallel interaction of work in the computer class and the instrumental performance class.

The observations during the experiment and individual interviews on the results of experimental training with the pianist students allowed to:

- reveal musical styles, timbres, and textures of performance, most adaptive to the sound specifics of electronic musical instruments;

- define the best ways to arrange keyboard electronic musical instruments and additional devices;

- make adjustments to the standard formats of creative and performing reporting of the students using the computer and music technologies;

- form exemplary combinations of repertoire and work material for the students in the instrumental performance and computer classes; and

- reveal effective combinations of electronic musical instruments, computer software, and sound equipment.

The data served as the basis for the creation of new disciplines for the education of the bachelor's and master's degree instrumental performance students of the Institute of Culture and Arts of the Moscow City University.

\section{Conclusion}

It is appropriate to implement such educational innovations based on pedagogical universities. Innovative methodical and technological elements of the pianist students' training can act as a systemic addition to the fundamental traditional principles of music education.

The combined learning of electronic keyboards and applied software training helps to accelerate the formation of the students' competence in both fields. The selected creative and performing tasks assigned to the students corresponded to the goals of experimental training and motivated the students to further professional improvement. 
In today's environment, the prospects for creating highly specialized areas of performing students' training based on the technological resources of the digital interface of musical instruments are seen as positive.

The task of forming the optimal volume of the musical repertoire of different complexity levels, building special training modules for bachelor's and master's degree programs requires further solutions.

The development of special technical standards to accompany the music performance of students based on computer and music technology and electronic musical instruments is required.

\section{References}

1. M.N. Dudin, V.V. Afanasev, I.V. Afanasieva, R.G. Rezakov, Amazonia Investiga 8(21), 674-687 (2019)

2. G.V. Ganshina, E.V. Babaeva, E.I. Medved, I.D. Levina, A.V. Kamenets, Humanities and Social Sciences Reviews 7 (6) 722-726 (2019)

3. A.V. Kondaurova, N.N. Surtaeva, V.V. Afanasev, O.A. Ivanova, R.G. Rezakov, Espacios 39(46), 19 (2018)

4. V. Afanasev, M. Vrazhnova, L. Shypovskaya, M. Nechaev, S. Frolova, Astra Salvensis 6(12), 193-209 (2018)

5. E.M. Akishina, I.M. Krasilnikov, E.S. Medkova, European Journal of Science and Theology 13(3), 195-203 (2017)

6. M.A. Antonova, E.B. Beregovaya, O.B. Ushakova, L.G. Pak, E.R. Saitbaeva, M.K. Musafirov, M.V. Olinder, O.E Savenko, Humanities and Social Sciences Reviews 7, 1120-1127 (2019)

7. O.V. Stukalova, V.O. Malaschenko, G.A. Cornilov, A.V. Sychenkova, E.B. Kozlova, A.I. Prokopyev, Humanities and Social Sciences Reviews 7(5), 731-737 (2019)

8. E.G. Artemova, E.A. Bodina, S.V. Pokrovskaya, N.N. Telysheva, International Journal of Advanced Biotechnology and Research 10(1), 219-223 (2019)

9. I.B. Gorbunova, A. Kameris, E.N. Bazhukova, International Journal of Recent Technology and Engineering 8(6), 3040-3045 (2020)

10. I.B. Gorbunova, International Journal of Advanced Science and Technology 29 (S6), 600-615 (2020)

11. I.M. Krasilnikov, Quality - Access to Success 19(165), 103-109 (2018)

12. I.B. Gorbunova, A. Kameris, International Journal of Recent Technology and Engineering 8(2S4), 913-918 (2019)

13. E.Y. Levina, E.A. Bodina, E.G. Artemova, A.V. Kiryakova, V.V. Ogorodnikova, E.G. Matvievskaya, T.N. Kriskovets, S.G. Kashina. University intellectual capital formation and development 7(4), 1100-1107 (2019)

14. L.L. Alekseeva, E.A. Sorokoumova, L.N. Vakhrusheva, A.V. Litvinov, N.V. Popovitskaya, O.G. Filenkova, Espacios 39(5), 28 (2018)

15. I.A. Belokon, L.A. Shibankova, A.V. Ignatieva, S.M. Kargapoltsev, E.A. Ganaeva, E.A. Beroeva, S.N. Trubenkova, E.B. Kozlova, Humanities and Social Sciences Reviews 4, 1061-1068 (2019)

16. E.I. Cherdymova, L.I. Ukolova, O.V. Gribkova, E.P. Kabkova, I.V. Kudrinskaya, L.I. Tararina, R.A. Kurbanov, A.M. Belyalova, Ekoloji 27(106), 541-546 (2018) 
17. E.I. Medved, O.I. Kiseleva, I.D. Levina, M.G. Kaytandzyan, G.I. Gribkova, Journal of advanced Research in Law and Economics 9(6), 2072-2078 (2018) 